\title{
Expanding the chemical repertoire of DNA nanomaterials generated by rolling circle amplification
}

Ysobel R. Baker ${ }^{1, \S}$, Liyiwen Yuan ${ }^{1, \S}$, Jinfeng Chen ${ }^{1}$, Roman Belle ${ }^{1}$, Robert Carlisle ${ }^{2}$, Afaf H. ElSagheer ${ }^{1,2}$ and Tom Brown ${ }^{1, *}$

${ }^{1}$ Department of Chemistry, University of Oxford, Oxford, OX1 3TA, UK

2 Institute of Biomedical Engineering, University of Oxford, Oxford, OX3 7DQ, UK

${ }^{3}$ Chemistry Branch Department of Science and Mathematics, Suez University, Suez 43721, Egypt

* To whom correspondence should be addressed. Email: tom.brown@chem.ox.ac.uk

$\S$ The authors wish it to be known that, in their opinion, the first two authors should be regarded as joint First Authors

\section{ABSTRACT}

Rolling circle amplification (RCA) is a powerful tool for the construction of DNA nanomaterials such as hydrogels, high-performance scaffolds and DNA nanoflowers (DNFs), hybrid materials formed of DNA and magnesium pyrophosphate. Such DNA nanomaterials have great potential in therapeutics, imaging, protein immobilisation, and drug delivery, yet limited chemistry is available to expand their functionality. Here, we present an orthogonal strategy to produce densely modified RCA products and DNFs. We show that it is possible to selectively functionalise the DNA component of these materials, their protein cargo, or both, thereby greatly expanding the chemical repertoire available to these systems. We then use this methodology to construct DNFs bearing multiple surface aptamers capable of binding to cancer cells that overexpress the HER2 oncobiomarker, demonstrating the therapeutic and diagnostic potential of this chemistry.

\section{INTRODUCTION}

DNA provides an excellent basis for the fabrication of novel nanomaterials (1), having many advantageous properties including biocompatibility, water solubility, structural programmability through Watson-Crick base pairing, and complementary target recognition. DNA-based nanomaterials are expected to have a major impact in the biomedical and diagnostic fields due to their high target specificity, extended half-life, and improved eukaryotic cell uptake (in general other biological macromolecules do not enter cells efficiently).

Depending on the desired properties of the DNA nanomaterials, the DNA component can be synthetic $(2,3)$, isolated from biological sources (e.g. bacteriophages) (4), or produced using amplification techniques such as the polymerase chain reaction (PCR) $(5,6)$ or rolling circle amplification (RCA) (7). These alternative approaches produce DNA on varying length scales and with different characteristics. Uniquely, RCA produces long concatamers of single stranded DNA (ssDNA) that can be assembled into a variety of nanomaterials (8) including nanoribbons (9), nanosprings (10), and templating nanoscaffolds (11). 
RCA can also be used to directly synthesise materials such as DNA nanoflowers (DNFs), DNAinorganic hybrid superstructures with flower-like morphology (Figure 1) (12). During RCA, a DNA polymerase (typically the highly processive polymerase phi29) repeatedly traverses a circular DNA template many many times, producing long ssDNA formed of repeating units which are complementary to the template. A pyrophosphate anion (PPi) is released with each dNTP incorporation, and these polyanions co-crystallise with both the $\mathrm{Mg}^{2+}$ cations present in the amplification buffer and the growing DNA chains to form hybrid nanostructures (13). Analogous to this, rolling circle transcription can be used to prepare similar material termed RNA microsponges, consisting of RNA, PPi, and $\mathrm{Mg}^{2+}$ ions(14). DNFs benefit from the favourable biological properties and programmability of DNA combined with the structure and stability associated with $\mathrm{Mg}_{2} \mathrm{P}_{2} \mathrm{O}_{7}$ complexation.

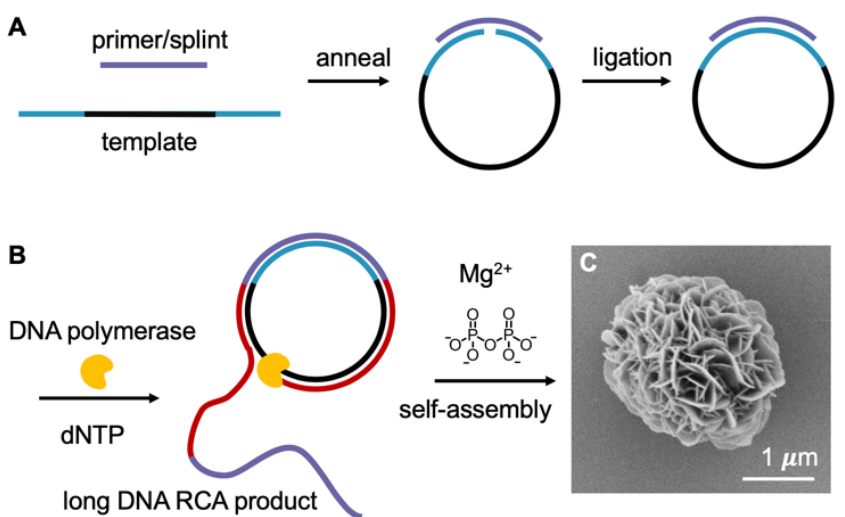

Figure 1. Enzymatic preparation of DNFs. (A) A linear DNA template with a $5^{\prime}$ phosphate is annealed to a DNA splint and cyclised using T4 DNA ligase. (B) The splint is extended using a DNA polymerase with strand displacement activity (yellow) to give long ssDNA formed of repeating units (red and purple). The DNA then co-crystallises with the pyrophosphate by-product and with $\mathrm{Mg}^{2+}$ ions present in the amplification buffer to give DNFs. (C) Scanning electron microscopy (SEM) image of resulting DNFs prepared using unmodified dNTPs.

DNFs have promise as therapeutic and diagnostic agents owing to their enhanced cellular uptake, cell targeting ability, and resistance to nuclease degradation $(12,15)$. Through modification of the circular template, DNFs can be programmed to contain specific functionality such as aptamers for cell targeting (12), immunostimulatory regions (16), DNAzymes for targeted RNA degradation (17), and drug loading sequences (15). The size of the particles can be controlled to some extent by altering the amplification time (15), adjusting the $\mathrm{Mg}^{2+}$ concentration (18), or by condensing the resulting particles (19). DNFs can be prepared that contain proteins, including enzymes for intracellular delivery and catalytic enhancement (20), and the inorganic core of the particles can be altered by changing the reaction buffer $(21,22)$. Despite these advances, there is limited chemistry available to modify the DNA component of these particles or to covalently attach extra functionality to the nanoconstructs.

The human epidermal growth factor receptor (HER2) is over expressed in $>20 \%$ of breast cancers and we wanted to develop DNFs capable of specifically binding to HER2 positive (HER2 ${ }^{+}$) cancer 
cells for therapeutic and diagnostic applications. Our initial attempts at targeting HER2 ${ }^{+}$cells using DNFs formed from a concatemer of a previously reported HER2 targeting aptamer (23) were unsuccessful, and resulted in low binding. We hypothesised that the aptamer was unable to form the required 3D structure within the DNF core to enable target engagement, and that having the aptamer decorating the surface would improve the interaction. Hence, we sought to develop a generic method to attach functionality to DNFs which would also be applicable to a vast array of emerging RCA fabricated nanomaterials and their applications.

Here we present a simple 2-step approach that utilises high concentrations of modified dNTPs ( $\mathrm{dN}^{*} \mathrm{TPs}$ ) with small chemical handles during the construction of the DNFs. As many RCA generated materials rely on Watson-Crick base pairing for function and structure we decided that a nonhydrogen bonding nucleobase face would be the best position to attach functionality. We demonstrate the effectiveness of this approach by DNF synthesis and show that the choice of modified nucleotide triphosphate and labelling conditions significantly affect the outcome of RCA and nature of the materials produced. This methodology can be used to attach a range of chemistries to the surface of the DNFs, and can also be used to modify the protein cargo of DNFs leaving the DNA component intact. Using this approach, we have prepared DNFs that target HER2 ${ }^{+}$cancer cells and discuss the importance of material design when using aptamers to interact with receptor proteins on cell surfaces.

\section{MATERIAL AND METHODS}

The general methods, protocols for the synthesis of oligonucleotides and cyclisation of templates, details for the preparation of each type of DNF described in the manuscript, and protocols for labelling are given in the Supplementary Information.

\section{RESULTS AND DISCUSSION}

\section{Overview of strategy used to generate chemically modified RCA nanomaterials}

An overview of our functionalisation strategy is given in Figure $2 \mathrm{~A}$. $\mathrm{dN}^{*} \mathrm{TPs}$ (Figure $2 \mathrm{~B}$ ) are added during RCA, generating long ssDNA with azide, alkyne and/or amine handles. The ssDNA cocrystallises with PPi and $\mathrm{Mg}^{2+}$ to give DNFs with chemical handles that can be used for post-synthetic functionalisation, either through copper catalysed alkyne-azide cycloaddition (CuAAC) $(24,25)$, strainpromoted alkyne-azide cycloaddition (SPAAC) (26) using a cyclooctyne such as BCN (bicyclo [6.1.0] non-4-yne)(27), or $\mathrm{N}$-hydroxysuccinimide (NHS) ester mediated amine labelling. $\mathrm{dN}^{*}$ TPs 1-5 are mimics of dTTP whereas 6 is a mimic of dCTP. By altering the ratio of modified to canonical dNTPs it is possible to control the degree of labelling, and using separate dCTP and dTTP analogues enables high levels of dual labelling within the same construct. $\mathrm{dN}^{*} \mathrm{TPs}$ 1-4 and 6 are commercially available, and in this study 1 (28), 3 (29), and 5 (30) were prepared according to literature procedures and converted to the corresponding sodium salts before use. $\mathrm{dN}^{*} \mathrm{TP} 3$ has been used with phi29 
previously (31). The sequences and cyclisation protocols of the oligonucleotides used in this study are described in the supporting information.

A
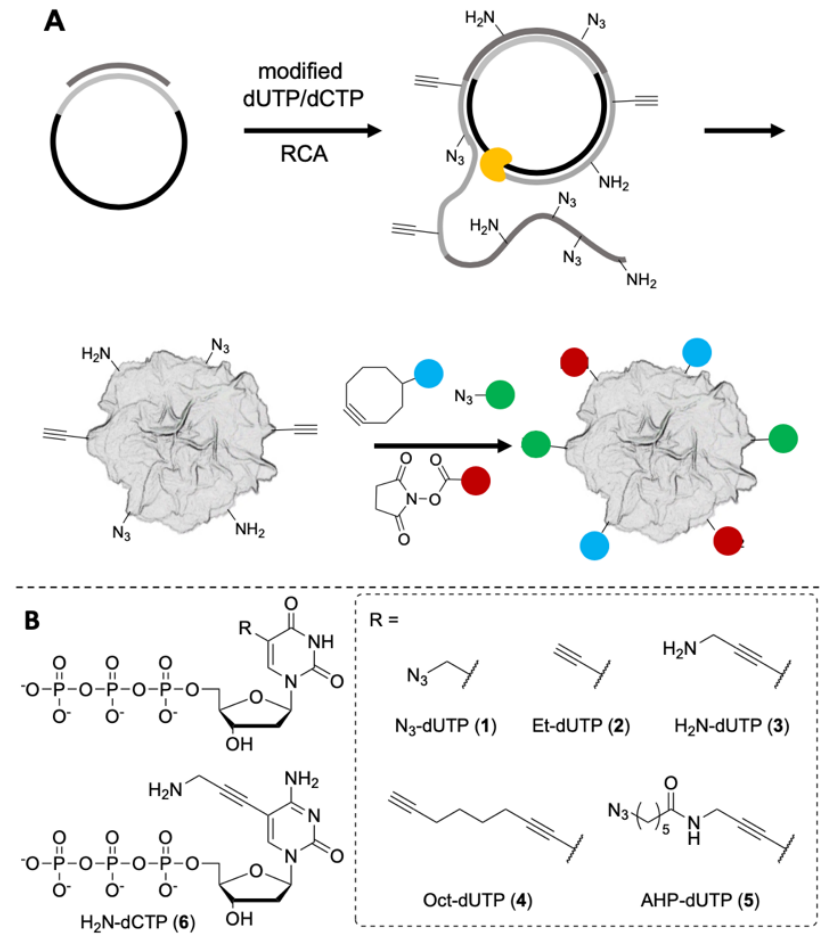

Figure 2. (A) Enzymatic preparation of functionalisable DNFs and subsequent orthogonal labelling. (B) $d N^{*}$ TPs investigated in this study.

\section{Identifying $\mathrm{dN}^{*} \mathrm{TPs}$ that do not perturb the structure or DNA component of RCA generated nanomaterials such as DNFs}

To achieve a high level of functionality in the DNFs we needed to identify $\mathrm{dN}^{*}$ TPs that could be incorporated to high degree without altering the DNF structure. Whilst $\mathrm{dN}^{*} \mathrm{TPs}$ have been used to add functionality to RCA products, phi29 does not incorporate bulky $\mathrm{dN}^{*} \mathrm{TPs}$ without a reduction in polymerase efficiency (31-35), and the standard approach in RCA is to introduce dN*TPs at low concentration in the reaction mixture (typically $40 \mu \mathrm{M}$ ) in the presence of a much higher concentration of the four canonical triphosphates (typically $2 \mathrm{mM}$ of each triphosphate) (36). This obviously gives very low labelling density.

We first looked at the effect of $\mathrm{dN}^{*}$ TPs 1-6 (Figure 2B) on the quantity and length of DNA produced and analysed the percentage of DNA incorporated into the DNFs using SYBR gold, a fluorescent dye that binds both single and double stranded DNA (Figure 3) (21). DNF synthesis was carried out substituting the canonical dNTP with varying percentages of $d N^{*} T P$ maintaining a total concentration of $2 \mathrm{mM}$. The total reaction volumes and concentration of all other components was kept the same, allowing direct comparisons. Following RCA, the DNFs were separated from the RCA mixture by centrifugation and the relative amounts of 'free' DNA (i.e. DNA not incorporated into DNFs) and DNA 
encapsulated in the DNFs were directly compared using SYBR gold. The length of DNA produced was also determined by agarose gel electrophoresis (Figure S1-6). Extended DNA was produced with all $\mathrm{dN}^{*} \mathrm{TPs}$ tested; however, the yields varied significantly. Complete substitution of dUTP with EtdUTP 2 resulted in only 14\% reduction in the total DNA produced (calculated by adding the fluorescence of the 'free' DNA to the value for the DNA within DNFs) and had little effect on the amount of DNA incorporated into the DNFs (Figure $3 \mathrm{~A}$ ). In contrast, substitution of dUTP with the larger alkyne Oct-dUTP 4 led to a $76 \%$ reduction in total DNA yield and a significantly reduced amount of DNA present in the DNFs (Figure 3B). A similar trend was observed with the azide triphosphates $\mathrm{N}_{3}$-dUTP 1 and the bulkier AHP-dUTP 5 (Figure $3 C$ and D), albeit to a smaller degree (16\% and $42 \%$ reduction in total DNA respectively), suggesting that phi29 tolerates smaller nucleobase modifications better than larger ones, which is in agreement with other reports (31-35). We hypothesise that the incorporation of Oct-dUTP 4 results in a particularly hydrophobic RCA product, and this precipitates/aggregates at high incorporation of $\mathbf{4}$, preventing further extension of the growing chain. Phi29 incorporated the amino modified dU and dC triphosphates 3 and $\mathbf{6}$ similarly (Figure 3E and F).

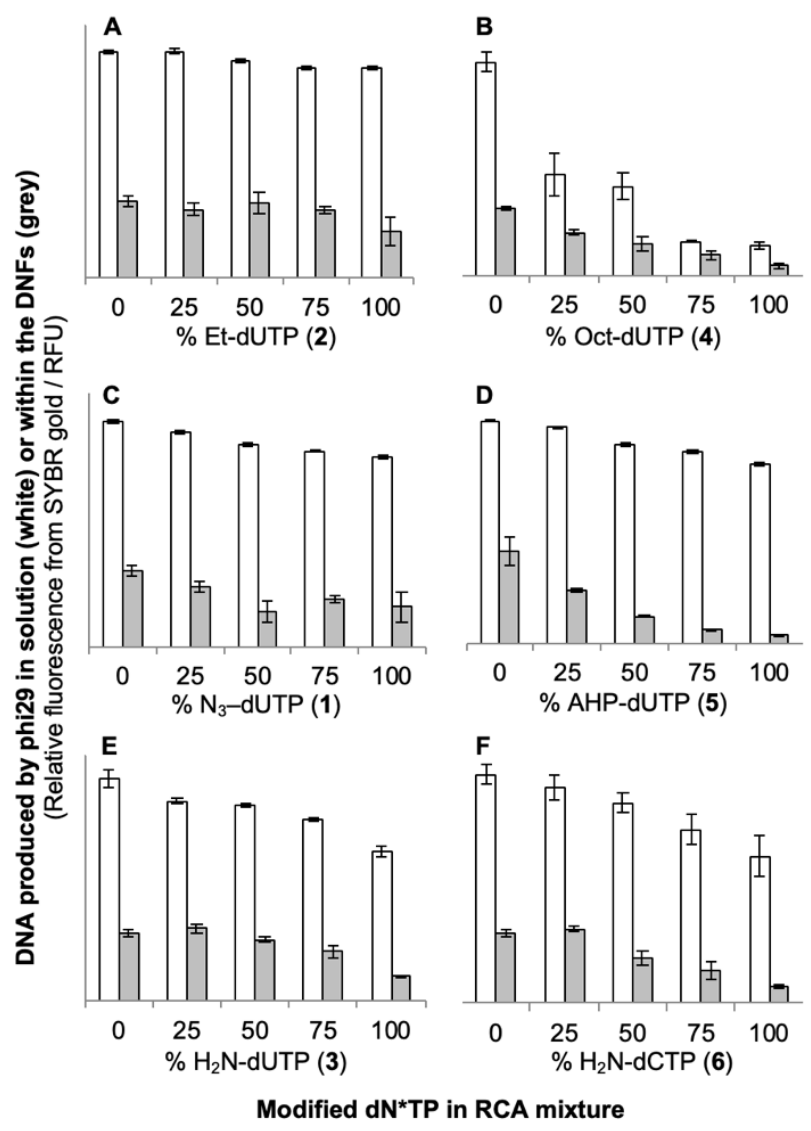

Figure 3. Fluorescence-based comparison of DNA produced during DNF synthesis when dUTP or dCTP were substituted with different percentages of each of the $\mathrm{dN}^{*}$ TPs in the amplification step. In all cases the total dNTP concentration was kept at $2 \mathrm{mM}$ and the $\% \mathrm{dN}^{*} T P$ is indicated on the x-axis. White bars represent the 'free' DNA and grey bars represent the DNA that is part of the DNFs. Ethylenediaminetetraacetic acid (EDTA) was added prior to incubation with SYBR gold to release the entrapped DNA. Error bars represent the standard deviation (SD) of three different RCA reactions. 
Scanning Electron Microscopy (SEM) was used to compare the impact of these modifications on DNF structure (Figure 4, and S7-13). Triphosphates 1 and 2 had a minimal effect on structure, whereas $\mathbf{4}$ caused the DNA to coagulate, and $\mathbf{5}$ resulted in much smaller particles, which correlates with the levels of DNA observed in the corresponding DNFs (grey bars, Figure 3). It is noteworthy that $\mathrm{dN}^{*}$ TPs 4 and 5 both have long hydrophobic chains in the 5-position of the nucleobase. The size of particles formed using increasing amounts of the amino modified dN*TPs 3 and $\mathbf{6}$ was inversely linked to the percentage of modified triphosphate used (Figure S10 and S13). This could be due to reduced DNA yields when using the amino $\mathrm{dN}^{*} \mathrm{TPs}$ and/or interaction of the cationic amine modifications with the anionic phosphodiesters in the DNA (35) (or the pyrophosphate component of the DNFs), leading to condensed structures.

The results shown in Figures 3 and 4 highlight the importance of understanding how dNTP modification can alter the properties of DNA and nanostructures generated during RCA. The choice of $d N^{*}$ TP clearly enables control over the size of the functionalisable DNFs which, given that the size of nanomaterials determines their fate in vivo (37), makes this a promising strategy when developing novel therapeutic agents. Having established that the $\mathrm{dN}^{*} \mathrm{TPs} 1,2,3$, and $\mathbf{6}$ could be used to prepare DNFs with little adverse effect on DNA produced, DNF structure, and DNF DNA content, we confirmed that each of the handles (azide, alkyne and amine) could subsequently react with chemical labels to functionalise the DNFs. Fluorescent dyes were used to provide a simple measurable property. 

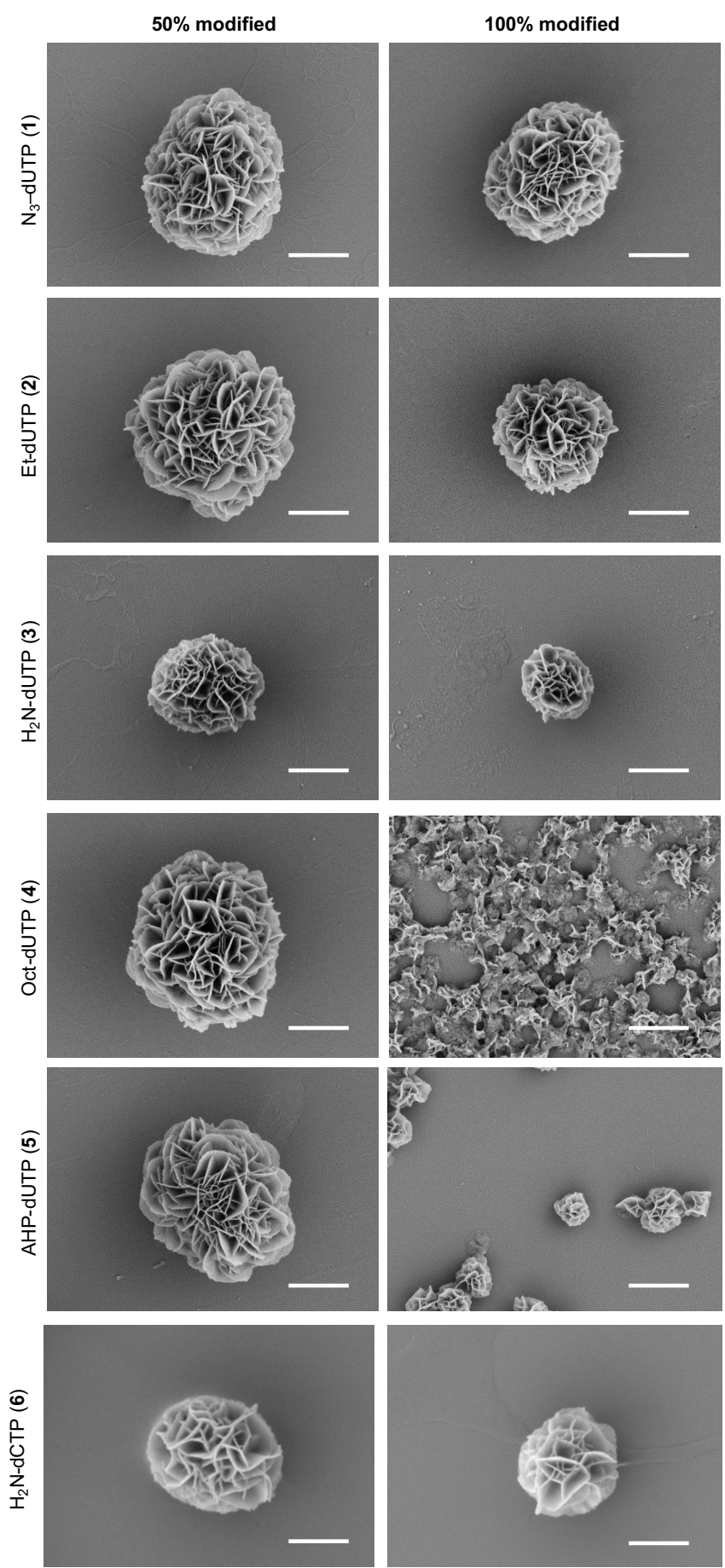

Figure 4. SEM images of DNFs produced with the different $\mathrm{dN}^{*} \mathrm{TPs}$. DNFs were prepared using 1:1 $\mathrm{dN}^{\star}$ TP to either dUTP or dCTP (50\% modified) or with complete replacement of the canonical dUTP (100\% modified). Figure 1C shows a typical unmodified DNF. Further images (low magnification, 25\% and $75 \%$ modified) are shown in the SI (Figure S7-13). Scale bars represent $1 \mu \mathrm{m}$. 


\section{Functionalisation of RCA generated DNA nanomaterials using orthogonal labelling chemistry}

NHS ester labelling. We first needed to identify a buffer for the functionalisation reactions that would not alter the DNF morphology or DNA content. Maintaining the pH between 7.2 and 9.0 is necessary for efficient NHS-ester labelling of amines, and as many organic labels have low solubility in water, we investigated the use of DMF, DMSO and MeCN as organic co-solvents. DNFs (unmodified) were incubated in different buffers (PBS, $10 \mathrm{mM} \mathrm{HEPES}$, and $10 \mathrm{mM}$ Tris), and we evaluated their stability by SEM imaging and agarose gel electrophoresis. Our preliminary results indicated that the best buffer for the labelling experiments is $10 \mathrm{mM}$ HEPES at pH 8 with $50 \%$ DMSO as the co-solvent at room temperature, and that it is necessary to include $10 \mathrm{mM} \mathrm{Mg}^{2+}$ to prevent the DNA from disassociating from the nanoflowers. In contrast, PBS led to increased loss of DNA from the DNFs (see Figure S14). This is an important observation given that many DNF protocols utilise PBS for DNF storage and/or fluorescence assisted cell sorting (FACS) experiments (15). $\mathrm{H}_{2} \mathrm{~N}$-DNFs prepared using $\mathrm{H}_{2} \mathrm{~N}$-dUTP 3 were treated with fluorescein-NHS (FAM-NHS) (Figure 5) in labelling buffer (50\% DMSO, $10 \mathrm{mM}$ HEPES, $\mathrm{pH} 8.0,10 \mathrm{mM} \mathrm{MgSO}_{4}$ ).
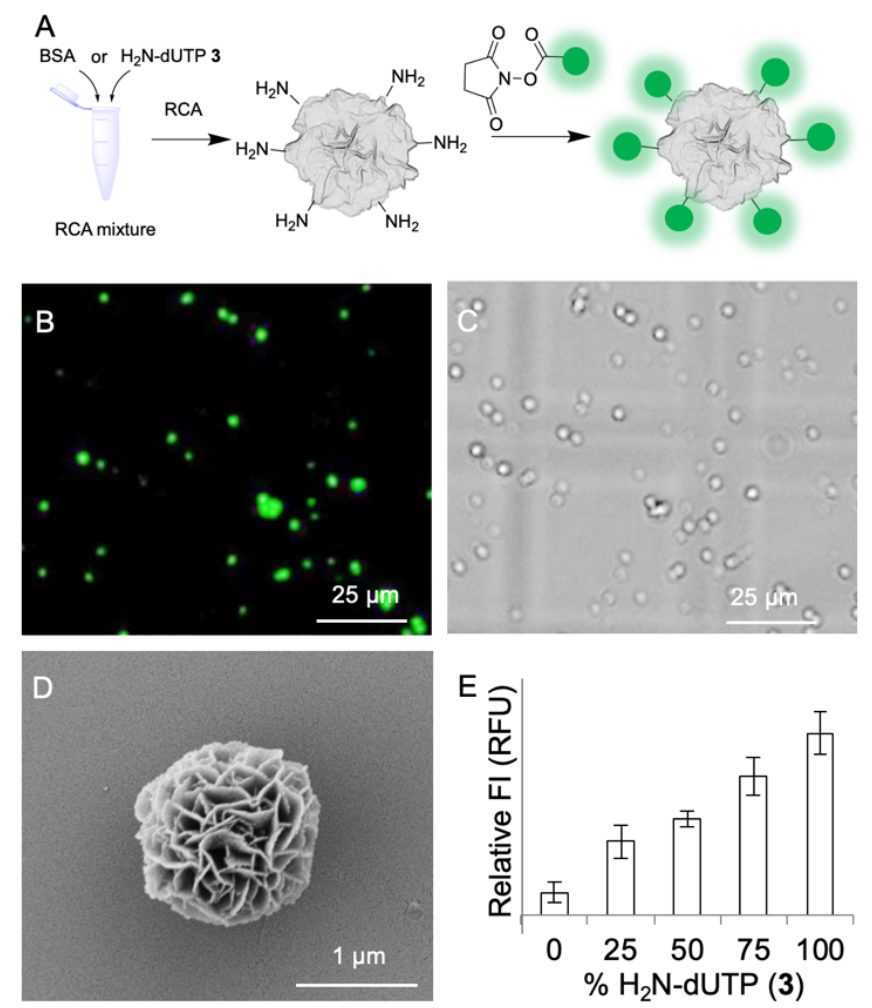

Figure 5. NHS ester labelling of $\mathrm{H}_{2} \mathrm{~N}$-DNFs. (A) Schematic of the method; (B) Fluorescence image of FAM functionalised $\mathrm{H}_{2} \mathrm{~N}$-DNFs under $475 \mathrm{~nm}$ excitation (prepared using $50 \% \mathrm{H}_{2} \mathrm{~N}$-dUTP 3; (C) Brightfield image of sample shown in $\mathrm{B}$. Some particle migration occurs as the samples are in water; (D) SEM image of FAM modified DNF prepared with $50 \% \mathrm{H}_{2} \mathrm{~N}$-dUTP 3; (E) Relative FAM fluorescence intensity $(\mathrm{FI})$ of DNFs containing varying levels of $\mathrm{H}_{2} \mathrm{~N}$-dUTP 3 and labelled with FAMNHS. Error bars represent the SD of three repeat reactions. 
Fluorescence microscopy showed punctate fluorescence in the relevant channel (Figure 5B and C) and SEM revealed that no structural perturbation occurred (Figure 5D). Next, we prepared $\mathrm{H}_{2} \mathrm{~N}-\mathrm{DNF}$ using different ratios of $\mathrm{H}_{2} \mathrm{~N}$-dUTP 3 to dTTP. The DNFs were collected by centrifugation, washed with water and labelled with FAM-NHS. The particles were then treated with EDTA to release the DNA and the relative fluorescence was measured and compared to an untreated sample. Whilst an increase in labelling occurred in comparison with the unmodified DNFs, non-specific labelling also occurs (Figure $5 E$ ). A small amount of phi29 polymerase is incorporated in the DNFs during RCA (20) and we hypothesise that the background labelling is occurring on the lysine amino groups of the enzyme. To test this, we used sodium dodecyl sulfate polyacrylamide gel electrophoresis (SDS-PAGE) to compare the proteins present in unmodified DNFs which had been subjected to the labelling conditions with phi29 that had been treated with the same NHS active ester. The results show that the non-specific labelling was not occurring on the DNA, but rather with the encapsulated phi29 protein (Figure S15). Expanding on this hypothesis we added varying quantities of bovine serum albumin (BSA), a lysine-rich protein which is known to be encapsulated into DNFs (20), during the RCA reaction. We then successfully labelled the particles with fluorescent dyes and analysed the resulting DNFs (see supplementary discussion). This indicates that further orthogonality within DNFs can be achieved by adding a lysine rich protein (phi29 alone does not result in a suitably high level of labelling) and allows the preparation of functionalised DNFs, where the DNA component remains unmodified. We envisage that this approach will find applications in the delivery of non-modified oligonucleotides for applications such as antisense or CRISPR-Cas9 gene editing.

SPAAC labelling. Azide modified DNFs ( $\mathrm{N}_{3}$-DNFs) were prepared using 50\% $\mathrm{N}_{3}$-dUTP 1 and $50 \%$ dTTP during the RCA reaction. Initial labelling attempts using Cy5-BCN proved unsuccessful, a fluorescent signal was observed for both modified and unmodified DNFs treated under the same conditions, suggesting that the hydrophobic dye was sticking non-specifically to the particles (Figure $\mathrm{S} 16)$. To avoid this the $\mathrm{N}_{3}$-DNFs were treated with the more polar fluorescein-BCN (FAM-BCN) in labelling buffer for $2 \mathrm{~h}$ (Figure 6A). The FAM labelled $\mathrm{N}_{3}$-DNFs showed absorption and emission spectra indicative of successful click labelling whilst the non-modified DNFs showed no observable fluorescence, confirming that non-specific FAM-labelling was not occurring (Figure S17). Fluorescence microscopy showed punctate fluorescence (Figure 6B), agarose gel electrophoresis indicated that the dye was successfully linked to the DNA component of the particles (Figure S18), and SEM confirmed that the labelling step did not alter DNF structure (Figure 6D). The degree of fluorescence labelling observed correlated with the concentration of $\mathrm{N}_{3}$-dUTP 1 used in the RCA reaction (Figure $6 \mathrm{E}$ ), demonstrating that this approach can be used to carefully control the degree of chemical modification in DNFs. Unlike with the NHS labelling experiments, no background labelling occurred, this is to be expected as none of the components of the RCA mixture should react with an alkyne. 

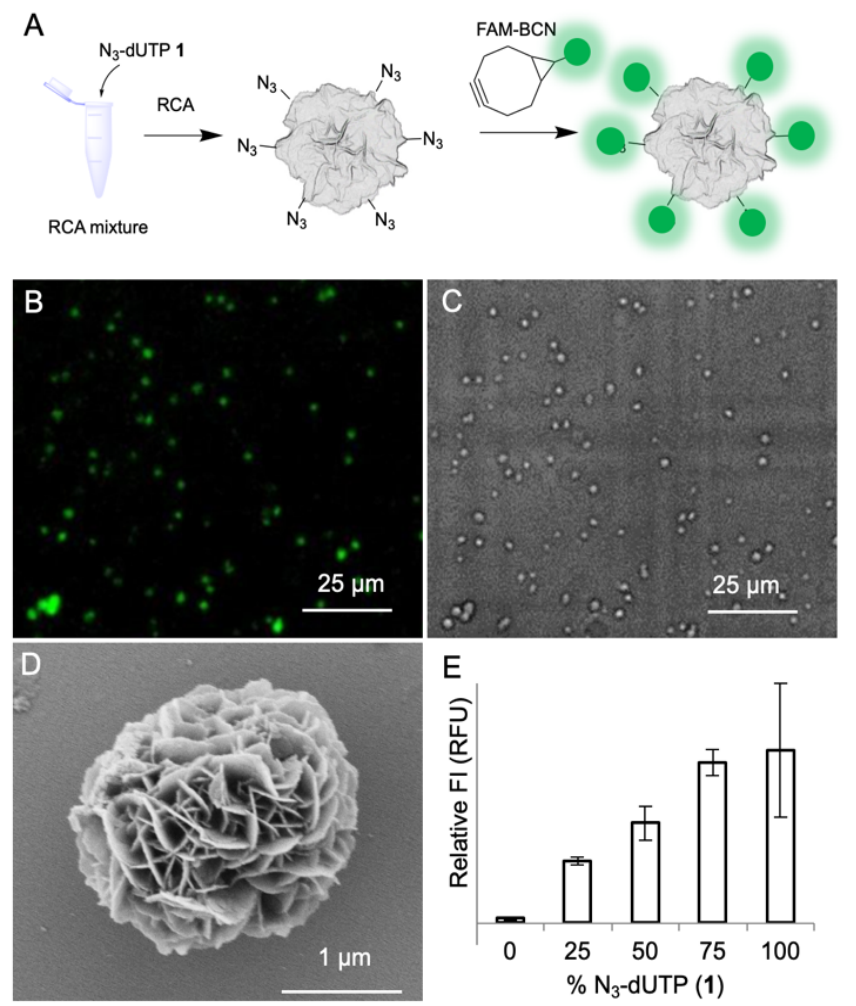

Figure 6. SPAAC labelling of $\mathrm{N}_{3}$-DNFs. (A) Schematic overview of methodology; (B) Fluorescence image of FAM functionalised $\mathrm{N}_{3}$-DNFs (prepared using 50\% $\mathrm{N}_{3}$-dUTP 1 under $475 \mathrm{~nm}$ excitation; (C) Brightfield image of sample shown in B. Some particle migration occurs as the samples are in water; (D) SEM images of FAM modified DNF prepared with 50\% N3-dUTP 1; (E) Relative FAM fluorescence observed for DNFs containing varying levels of $\mathrm{N}_{3}$-dUTP 1 and labelled with FAM-BCN. Error bars represent the SD of three repeat reactions.

CUAAC labelling. Cu-catalysed click labelling of alkyne functionalised DNFs (=-DNFs) prepared using Et-dUTP 2 and labelled with 3-azido-7-hydroxycoumarin ( $\mathrm{N}_{3}$-coumarin), a weakly-fluorescent dye that becomes highly fluorescent following 1,3-cycloaddition to an alkyne to form a triazole, gave similar results (Figure 7). Here, $\equiv$-DNFs were incubated with $\mathrm{N}_{3}$-coumarin, $\mathrm{CuSO}_{4}$, trishydroxypropyltriazolylmethylamine catalyst (THPTA) and sodium ascorbate reductant (NaAsc) for $2 \mathrm{~h}$ in labelling buffer. As expected the dye was 'turned-on' by the reaction proportionally to the amount of 2 added during RCA (Figure 7B), confirming that covalent labelling was occurring via CuAAC chemistry. We also observed that untreated DNFs show emission at around $500 \mathrm{~nm}$ when excited at $410 \mathrm{~nm}$. Whilst we do not have an explanation for this, it does account for the high relative e background fluorescence observed for the sample prepared without $\mathrm{N}_{3}$-dUTP 1. 

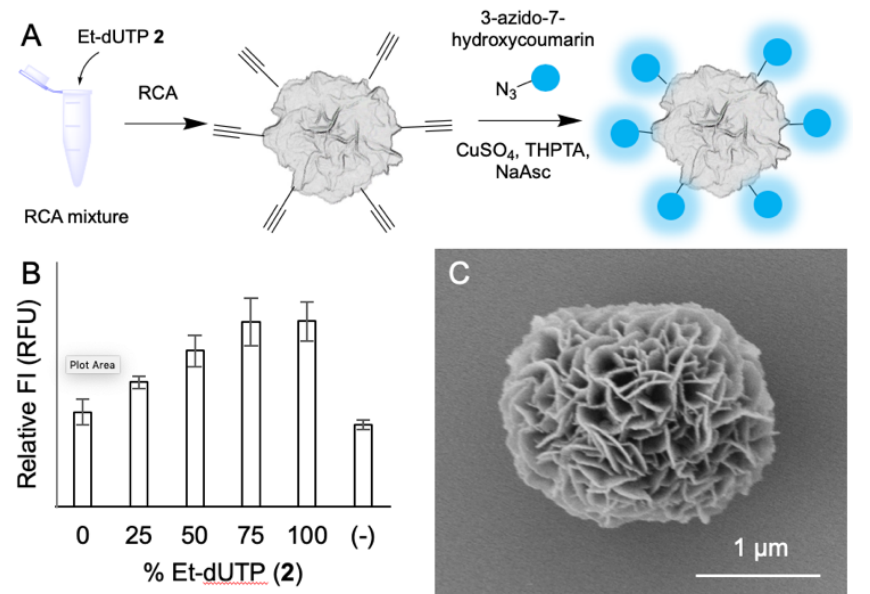

Figure 7. CuAAC labelling of $\equiv-D N F s$. (A) Schematic overview of the methodology; (B) Relative coumarin fluorescence (Excitation: 404-15 nm, Emission: 477-20 nm) of $\equiv-D N F s$ prepared with varying amounts of Et-dUTP 2 after CuAAC click-labelling with $\mathrm{N}_{3}$-coumarin. Error bars represent the SD of three repeat reactions, (-) is non-treated DNFs. (C) SEM image of an $\equiv$-DNF prepared with $100 \%$ Et-dUTP 2, and labelled with $\mathrm{N}_{3}$-coumarin.

\section{Using a combination of $d N^{*} T P s$ to attach multiple functionality to DNFs}

The methods described above can also be used to add more than one function to DNFs through dual labelling of the same construct. Specifically, two different $\mathrm{dN}^{*} \mathrm{TPs}$, or a $\mathrm{dN}^{*} \mathrm{TP}$ and a lysine rich protein (i.e. BSA) are added to the RCA mixture, producing DNFs with azide or alkyne groups for click and also amines for NHS labelling of the same construct. To show this we labelled DNFs prepared using 25\% $\mathrm{N}_{3}$-dUTP 1 and 25\% $\mathrm{H}_{2} \mathrm{~N}$-dCTP 6 and simultaneously labelled with TAMRA-NHS and FAM-BCN (Figure S19). To demonstrate the versatility of this approach, we also attached a fluorescently labelled peptide (Figure 8). The peptide was synthesised with both a TAMRA dye for visualisation and an N-terminal azide (Figure S20). DNFs were prepared using both $\mathrm{H}_{2} \mathrm{~N}$-dCTP 6 and Et-dUTP 2 in the RCA reaction mixture, yielding DNFs with amine and alkyne handles. These were treated with FAM-NHS and the azide-functionalised peptide in labelling buffer containing $\mathrm{CuSO}_{4}$, THPTA, and NaAsc. SEM imaging indicated no perturbation in structure (Figure 8E), and fluorescence microscopy demonstrated that labelling had successfully occurred (Figure 8B-D). 

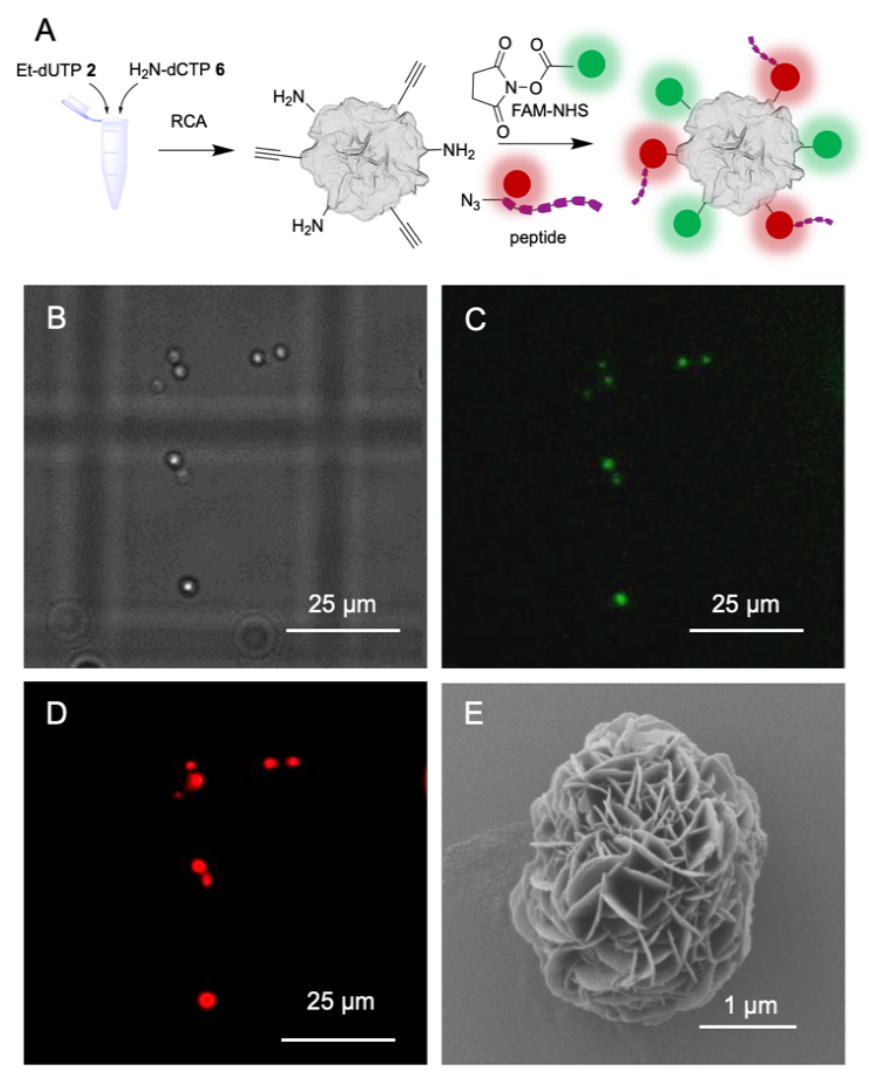

Figure 8. Dual labelling of DNFs prepared using 25\% Et-dUTP 2 and $25 \% \mathrm{H}_{2} \mathrm{~N}$-dCTP 6 then simultaneously functionalised with an azide/TAMRA modified peptide and FAM-NHS (A) Schematic of the method; (B) Brightfield image of dual labelled DNFs; (C) Fluorescence image under $475 \mathrm{~nm}$ excitation confirming attachment of FAM; (D) Fluorescence image under $542 \mathrm{~nm}$ excitation confirming attachment of the TAMRA peptide; (E) SEM image of dual labelled construct.

\section{Construction of HER2 targeting nanomaterials}

To demonstrate the diagnostic and therapeutic applications of these nanomaterials, we used our labelling approach to functionalise the surface of DNFs with a HER2 binding aptamer (23) and compared their ability to bind SKBR-3 HER2 ${ }^{+}$cells with DNFs incorporating the same aptamer which was prepared using the conventional pure RCA-based approach. The binding of the HER2 aptamer to SKBR-3 $\left(\right.$ HER2 $\left.^{+}\right)$cells has been well characterised previously $(38,39)$, providing a good model system for this study. Conventional aptamer-DNFs (cApt-DNFs), where the DNFs are formed from a concatemer of the HER2 aptamer, were prepared using $\mathrm{N}_{3}$-dUTP 1 and a cyclic template which encodes for the aptamer during the RCA step, and subsequently labelled with FAM-BCN. Functionalised aptamer-DNFs (fApt-DNFs) were prepared using the CuAAC labelling methodology as follows. A circular template with a randomly generated 'non-aptamer' sequence and $\mathrm{N}_{3}$-dUTP 1 were used to prepare non-aptamer $\mathrm{N}_{3}$-DNFs. These were then functionalised with the HER2 aptamer (synthesised with both FAM and alkyne moieties) using CuAAC labelling, and further treated with FAM-BCN to enhance their fluorescence (see Figure S21). SEM confirmed the CuAAC labelling step did not perturb DNF structure (Figure S22). Control-DNFs were also prepared, where the 'non- 
aptamer' DNFs were labelled with FAM-BCN and not the aptamer. The binding of these DNFs to SKBR-3 $\left(\mathrm{HER}^{+}\right.$) cells was evaluated by flow cytometry. fApt-DNFs showed higher binding than both the cApt-DNFs and control-DNFs (Figure 9A), indicating attachment of aptamers on the surface of DNFs improves their recognition and binding to the SKBR-3 cells. It is likely that that post-synthesis labelling with HER2 aptamers avoids extensive intermolecular interactions between aptamers, maintains their complementary shape to receptors and ensures their presentation on the surface of the DNFs.

A key advantage of the functionalisation approach is that it enables the attachment of alternative non-nucleic acid chemistries, including peptides. To demonstrate a potential application, we attached a FAM-labelled analogue of a HER2 specific peptide A9 (40) (Figure S23) to the surface of the DNFs using CuAAC chemistry, generating peptide-modified DNFs (pept-DNFs). Here the peptide was functionalised with an azide and the DNFs were prepared using the random template and Et-dUTP 2. Flow cytometry suggested that the pept-DNFs have a similar binding affinity towards SKBR-3 cells as fApt-DNFs (Figure 9B). Interestingly, the peptide alone only showed weak affinity for the cells; however, when immobilised on the surface of DNFs significant binding occurred suggesting that multivalency is an important beneficial parameter. Investigations into the origin of these effects and the binding interactions between the cells and the DNFs are ongoing and beyond the scope of this study. Together, these observations highlight the importance of nanomaterial design and construction when working with different aptamers and ligands.

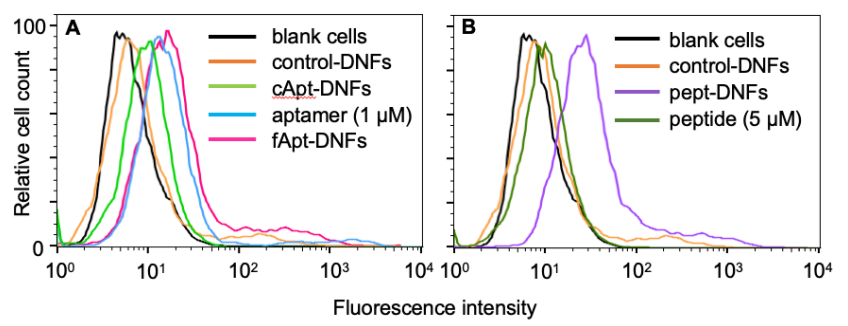

Figure 9. (A) Flow cytometry studies comparing the binding of DNFs prepared using the conventional approach (cApt-DNFs) with DNFs that have been functionalised using our labelling method (fAptDNFs) to SKBR-3, a HER2 ${ }^{+}$cell line. These results demonstrate that chemically attaching this aptamer to the surface of DNFs (fApt-DNFs) results in higher binding than when the same aptamer is templated into the DNF (cApt-DNFs); (B) Flow cytometry studies showing that DNFs functionalised with a HER2 binding peptide (pept-DNFs) also successfully bind to HER2 ${ }^{+}$SKBR-3 cells. This experiment was repeated and gave the same trends.

\section{CONCLUSION}

RCA-generated nanostructures and materials such as DNFs have great potential as practical scaffolds in the generation of complex hierarchical materials and nano-assemblies, especially if achieved through covalent attachment of functional components, within or on the surface of the materials. Here we have reported a post-assembly functionalisation method that has the potential to 
greatly expand the chemistry available to RCA-generated nanomaterials. We demonstrate that chemically-modified nucleoside triphosphates with small reactive handles can be added during RCA without significantly impairing the length or quantity of ssDNA and DNFs produced. Using DNFs, we show that these chemical handles can then be modified under mild conditions that do not alter the structural properties of the resulting nanomaterials. Our approach allows the covalent attachment of functional moieties that are non-nucleic acid in nature, thereby expanding the potential applications of these systems. A significant advantage is that this methodology makes use of commercially available $\mathrm{dN}^{*} \mathrm{TP}$ building blocks, avoiding the need for multistep organic synthesis. Many biologically relevant labels are already available with attached alkyne or azide functionality, or can be readily prepared, further expanding the versatility of this approach.

\section{ACKNOWLEDGEMENT}

The authors would also like to thank ATDBio Ltd. for use of equipment and financial support to J.C., and Professor Akane Kawamura for use of the peptide synthesiser.

\section{FUNDING}

This work was supported by the UK Engineering and Physical Sciences Research Council [grant numbers EP/R511742/1 to Y.B. and T.B, EP/M50659X/1 to R.B, EP/L024012/1 to R.C., EP/L003376/1]; Leverhulme Trust [grant number RPG-2015-005 to Y.B. and T.B.]; UK Biotechnology and Biological Sciences Research Council [grant numbers BB/J001694/2, BB/ R008655/1 to A.H.E-S and T.B.]; and a Chinese Scholarship Council-Oxford Scholarship to L.Y.

\section{REFERENCES}

1. Li, Z., Wang, J., Li, Y., Liu, X. and Yuan, Q. (2018) Self-assembled DNA nanomaterials with highly programmed structures and functions. Mater. Chem. Front., 2, 423-436.

2. Winfree, E., Liu, F., Wenzler, L.A. and Seeman, N.C. (1998) Design and self-assembly of twodimensional DNA crystals. Nature, 394, 539-544.

3. Wei, B., Dai, M. and Yin, P. (2012) Complex shapes self-assembled from single-stranded DNA tiles. Nature, 485, 623-626.

4. Rothemund, P.W.K. (2006) Folding DNA to create nanoscale shapes and patterns. Nature, 440, 297-302.

5. Han, D., Qi, X., Myhrvold, C., Wang, B., Dai, M., Jiang, S., Bates, M., Liu, Y., An, B., Zhang, F. et al. (2017) Single-stranded DNA and RNA origami. Science, 358, eaao2648.

6. Chidchob, P. and Sleiman, H.F. (2018) Recent advances in DNA nanotechnology. Curr. Opin. Chem. Biol., 46, 63-70.

7. Li, J., Lin, L., Yu, J., Zhai, S., Liu, G. and Tian, L. (2019) Fabrication and Biomedical Applications of "Polymer-Like" Nucleic Acids Enzymatically Produced by Rolling Circle Amplification. ACS Applied Bio Materials, 2, 4106-4120.

8. $\quad$ Ali, M.M., Li, F., Zhang, Z., Zhang, K., Kang, D.-K., Ankrum, J.A., Le, X.C. and Zhao, W. (2014) Rolling circle amplification: a versatile tool for chemical biology, materials science and medicine. Chem. Soc. Rev., 43, 3324-3341. 
9. Ma, Y., Zheng, H., Wang, C., Yan, Q., Chao, J., Fan, C. and Xiao, S.-J. (2013) RCA Strands as Scaffolds To Create Nanoscale Shapes by a Few Staple Strands. J. Am. Chem. Soc., 135, 29592962.

10. Zhang, K., Deng, R., Sun, Y., Zhang, L. and Li, J. (2017) Reversible control of cell membrane receptor function using DNA nano-spring multivalent ligands. Chem. Sci., 8, 7098-7105.

11. Deng, Z., Tian, Y., Lee, S.-H., Ribbe, A.E. and Mao, C. (2005) DNA-Encoded Self-Assembly of Gold Nanoparticles into One-Dimensional Arrays. Angew. Chem. Int. Ed., 44, 3582-3585.

12. Zhu, G., Hu, R., Zhao, Z., Chen, Z., Zhang, X. and Tan, W. (2013) Noncanonical Self-Assembly of Multifunctional DNA Nanoflowers for Biomedical Applications. J. Am. Chem. Soc., 135, 16438-16445.

13. Shopsowitz, K.E., Roh, Y.H., Deng, Z.J., Morton, S.W. and Hammond, P.T. (2014) RNAiMicrosponges Form through Self-Assembly of the Organic and Inorganic Products of Transcription. Small, 10, 1623-1633.

14. Lee, J.B., Hong, J., Bonner, D.K., Poon, Z. and Hammond, P.T. (2012) Self-assembled RNA interference microsponges for efficient siRNA delivery. Nat. Mater., 11, 316-322.

15. Lv, Y., Hu, R., Zhu, G., Zhang, X., Mei, L., Liu, Q., Qiu, L., Wu, C. and Tan, W. (2015) Preparation and biomedical applications of programmable and multifunctional DNA nanoflowers. Nat. Protoc., 10, 1508-1524.

16. Zhang, L., Zhu, G., Mei, L., Wu, C., Qiu, L., Cui, C., Liu, Y., Teng, I.T. and Tan, W. (2015) SelfAssembled DNA Immunonanoflowers as Multivalent CpG Nanoagents. ACS Appl. Mater. Interfaces, 7, 24069-24074.

17. Jin, Y., Li, Z., Liu, H., Chen, S., Wang, F., Wang, L., Li, N., Ge, K., Yang, X., Liang, X.-J. et al. (2017) Biodegradable, multifunctional DNAzyme nanoflowers for enhanced cancer therapy. NPG Asia Mater., 9, e365-e365.

18. Chen, J., Baker, Y.R., Brown, A., El-Sagheer, A.H. and Brown, T. (2018) Enzyme-free synthesis of cyclic single-stranded DNA constructs containing a single triazole, amide or phosphoramidate backbone linkage and their use as templates for rolling circle amplification and nanoflower formation. Chem. Sci., 9, 8110-8120.

19. Roh, Y.H., Lee, J.B., Shopsowitz, K.E., Dreaden, E.C., Morton, S.W., Poon, Z., Hong, J., Yamin, I., Bonner, D.K. and Hammond, P.T. (2014) Layer-by-Layer Assembled Antisense DNA Microsponge Particles for Efficient Delivery of Cancer Therapeutics. ACS Nano, 8, 9767-9780.

20. Kim, E., Zwi-Dantsis, L., Reznikov, N., Hansel, C.S., Agarwal, S. and Stevens, M.M. (2017) OnePot Synthesis of Multiple Protein-Encapsulated DNA Flowers and Their Application in Intracellular Protein Delivery. Adv. Mater., 29, 1701086.

21. Baker, Y.R., Chen, J., Brown, J., El-Sagheer, A.H., Wiseman, P., Johnson, E., Goddard, P. and Brown, T. (2018) Preparation and characterization of manganese, cobalt and zinc DNA nanoflowers with tuneable morphology, DNA content and size. Nucleic Acids Res., 46, 74957505.

22. Lee, J.S., Kim, H., Jo, C., Jeong, J., Ko, J., Han, S., Lee, M.S., Lee, H.-Y., Han, J.W., Lee, J. et al. (2017) Enzyme-Driven Hasselback-Like DNA-Based Inorganic Superstructures. Adv. Funct. Mater., 27, 1704213.

23. Mahlknecht, G., Maron, R., Mancini, M., Schechter, B., Sela, M. and Yarden, Y. (2013) Aptamer to ErbB-2/HER2 enhances degradation of the target and inhibits tumorigenic growth. Proc. Natl. Acad. Sci. USA, 110, 8170-8175.

24. Rostovtsev, V.V., Green, L.G., Fokin, V.V. and Sharpless, K.B. (2002) A Stepwise Huisgen Cycloaddition Process: Copper(I)-Catalyzed Regioselective "Ligation" of Azides and Terminal Alkynes. Angew. Chem. Int. Ed., 41, 2596-2599.

25. Tornøe, C.W., Christensen, C. and Meldal, M. (2002) Peptidotriazoles on Solid Phase: [1,2,3]Triazoles by Regiospecific Copper(I)-Catalyzed 1,3-Dipolar Cycloadditions of Terminal Alkynes to Azides. J. Org. Chem., 67, 3057-3064. 
26. Neef, A.B. and Luedtke, N.W. (2014) An Azide-Modified Nucleoside for Metabolic Labeling of DNA. ChemBioChem, 15, 789-793.

27. Dommerholt, J., Schmidt, S., Temming, R., Hendriks, L.J.A., Rutjes, F.P.J.T., van Hest, J.C.M., Lefeber, D.J., Friedl, P. and van Delft, F.L. (2010) Readily Accessible Bicyclononynes for Bioorthogonal Labeling and Three-Dimensional Imaging of Living Cells. Angew. Chem. Int. Ed., 49, 9422-9425.

28. Ren, X., El-Sagheer, A.H. and Brown, T. (2015) Azide and trans-cyclooctene dUTPs: incorporation into DNA probes and fluorescent click-labelling. Analyst, 140, 2671-2678.

29. Kore, A.R., Senthilvelan, A. and Shanmugasundaram, M. (2015) Highly Regioselective C-5 lodination of Pyrimidine Nucleotides and Subsequent Chemoselective Sonogashira Coupling with Propargylamine. Nucleosides, Nucleotides and Nucleic Acids, 34, 92-102.

30. Ren, X., El-Sagheer, A.H. and Brown, T. (2016) Efficient enzymatic synthesis and dual-colour fluorescent labelling of DNA probes using long chain azido-dUTP and BCN dyes. Nucleic Acids Res., 44, e79-e79.

31. Kim, K.-R., Röthlisberger, P., Kang, S.J., Nam, K., Lee, S., Hollenstein, M. and Ahn, D.-R. (2018) Shaping Rolling Circle Amplification Products into DNA Nanoparticles by Incorporation of Modified Nucleotides and Their Application to In Vitro and In Vivo Delivery of a Photosensitizer. Molecules, 23, 1833.

32. Smolina, I.V., Cherny, D.I., Nietupski, R.M., Beals, T., Smith, J.H., Lane, D.J., Broude, N.E. and Demidov, V.V. (2005) High-density fluorescently labeled rolling-circle amplicons for DNA diagnostics. Anal Biochem., 347, 152-155.

33. Linck, L., Reiß, E., Bier, F. and Resch-Genger, U. (2012) Direct labeling rolling circle amplification as a straightforward signal amplification technique for biodetection formats. Anal. Methods, 4, 1215-1220.

34. Yao, J., Flack, K., Ding, L. and Zhong, W. (2013) Tagging the rolling circle products with nanocrystal clusters for cascade signal increase in the detection of miRNA. Analyst, 138, 3121-3125.

35. Lee, S.Y., Kim, K.-R., Bang, D., Bae, S.W., Kim, H.J. and Ahn, D.-R. (2016) Biophysical and chemical handles to control the size of DNA nanoparticles produced by rolling circle amplification. Biomater. Sci., 4, 1314-1317.

36. Han, S., Lee, J.S. and Lee, J.B. (2017) Synthesis of a multi-functional DNA nanosphere barcode system for direct cell detection. Nanoscale, 9, 14094-14102.

37. Hoshyar, N., Gray, S., Han, H. and Bao, G. (2016) The effect of nanoparticle size on in vivo pharmacokinetics and cellular interaction. Nanomedicine (Lond), 11, 673-692.

38. Lee, H., Dam, D.H.M., Ha, J.W., Yue, J. and Odom, T.W. (2015) Enhanced Human Epidermal Growth Factor Receptor 2 Degradation in Breast Cancer Cells by Lysosome-Targeting Gold Nanoconstructs. ACS Nano, 9, 9859-9867.

39. Shen, Y., Zhang, J., Hao, W., Wang, T., Liu, J., Xie, Y., Xu, S. and Liu, H. (2018) Copolymer micelles function as $\mathrm{pH}$-responsive nanocarriers to enhance the cytotoxicity of a HER2 aptamer in HER2-positive breast cancer cells. Int. J. Nanomedicine, 13, 537-553.

40. Calce, E., Monfregola, L., Sandomenico, A., Saviano, M. and De Luca, S. (2013) Fluorescence study for selecting specific ligands toward HER2 receptor: an example of receptor fragment approach. Eur. J. Med. Chem., 61, 116-121. 